\title{
Hypertensive patients' preferences for complementary and alternative medicine and the influence of these preferences on the adherence to prescribed medication
}

\begin{abstract}
Purpose: This study aimed to understand hypertensive patients' perceptions of and adherence to prescribed medication.

Methods: A qualitative research study based on 23 purposely selected participants from a community health clinic in Malaysia. The participants underwent in-depth semi-structured interviews, and the data were analyzed using qualitative content analysis method.

Results: The participants were presented with six types of perceptions of medication. The majority of the participants had negative perceptions of Western medicine (WM), selfadjusted their prescribed medication with complementary and alternative medicine (CAM) and concealed their self-adjusting habits from their doctors. Participants who thought positively of WM took their prescribed medication regularly. Most of the participants perceived the nature of WM as not being curative because of its side effects. Patients have the right to choose their preferred medication when they understand their illness.

Conclusion: Local health care systems should provide patients with alternative health services that suit their requests.
\end{abstract}

Keyword: Adherence; Complementary and alternative medicine; Hypertension; Qualitative research 